Vol. 42 (1990) [511-516]

\title{
SOME PROPERTIES OF THE HAUSDORFF DISTANCE IN METRIC SPACES
}

\author{
Józef Banaś and Antonio Martinón
}

\begin{abstract}
Some properties of the Hausdorff distance in complete metric spaces are discussed. Results obtained in this paper explain ideas used in the theory of measures of noncompactness.
\end{abstract}

\section{INTRODUCTION}

The aim of this paper is to present some properties of the Hausdorff distance in complete metric spaces which are especially useful in the theory of measures of noncompactness.

Our considerations are closely related to measures of noncompactness defined in an axiomatic way (see $[1,2,3,4,6,7]$, for example). More precisely, we are interested in the following problem: Let $\mathcal{Z}$ be a subfamily of the family $\mathcal{M}$ of all nonempty and bounded subsets of a metric space. For $X \in \mathcal{M}$ define the number $H_{\mathcal{Z}}(X)$ as the Hausdorff distance of $X$ from $\mathcal{Z}$. What can we say about properties of the function $\boldsymbol{H}_{\mathcal{Z}}$ if we assume that the family $\mathcal{Z}$ satisfies some ordered or topological conditions?

Our results obtained here explain some ideas used in axiomatic definitions of measures of noncompactness proposed up to now. Particularly, we provide short proofs of a few theorems from the book [2] which are formulated here in a more general setting.

\section{NOTATION}

Let $(M, \rho)$ be a complete metric space.

By $\mathcal{M}_{M}$ (or, briefly $\mathcal{M}$ ) we shall denote the family of all nonempty and bounded subsets of $M$. Moreover, the family of all nonempty and relatively compact subsets of $M$ will be denoted by $\mathcal{N}$.

If $x \in M$ and $r>0$ then $K(x, r)$ will denote the open ball centred at $x$ and with radius $r$. Similarly, if $X \in \mathcal{M}$ then $K(X, r)$ denotes the ball having centre at $X$ and radius $r$ :

$$
K(X, r)=\bigcup_{x \in X} K(x, r)
$$

Received 15th January 1990

Copyright Clearance Centre, Inc. Serial-fee code: 0004-9729/90 SA2.00+0.00. 
The symbol $\bar{X}$ denotes the closure of a subset $X$ of $M$. Apart from this if $\mathcal{U}$ is an arbitrary family of subsets $X$ of $M$ then we define $\mathcal{U}^{c}$ as

$$
\mathcal{U}^{c}=\{X \in \mathcal{U}: X=\bar{X}\}
$$

Now, let $X, Y \in \mathcal{M}$. Denote

$$
\begin{gathered}
d(X, Y)=\inf \{r: X \subset K(Y, r)\} \\
D(X, Y)=\max \{d(X, Y), d(Y, X)\}
\end{gathered}
$$

The function $D(X, Y)$ is called the Hausdorff distance between sets $X$ and $Y$. It is well known that $D$ is a pseudometric on $\mathcal{M}$ and it is a complete metric on $\mathcal{M}^{c}$. Moreover, $\mathcal{N}^{c}$ forms a closed subspace of $\mathcal{M}^{c}$ with respect to the topology generated by $D[\mathbf{5}]$.

If $\mathcal{Z}$ is a nonempty subfamily of $\mathcal{M}$ then we will use the following notation:

$$
\begin{gathered}
D(X, \mathcal{Z})=\inf \{D(X, Z): Z \in \mathcal{Z}\} \\
d(X, \mathcal{Z})=\inf \{d(X, Z): Z \in \mathcal{Z}\}
\end{gathered}
$$

In what follows we shall consider the function $H_{\mathcal{Z}}: \mathcal{M} \rightarrow[0, \infty)$ defined in the following way

$$
H_{\mathcal{Z}}(X)=D(X, \mathcal{Z})
$$

where $\mathcal{Z}$ is the same as above. For brevity, we write $H(X)$ instead of $H_{\mathcal{Z}}(X)$.

\section{MaIN Results}

We start with the following simple but useful lemma.

Lemma 1. Let $A, B \in \mathcal{M}$ and $r>0$. If $B \subset K(A, r)$ then $A \cap K(B, r) \neq \emptyset$ and $B \subset K(A \cap K(B, r), r)$.

Proof: Take an arbitrary $b \in B$. By the assumption there exists $a \in A$ such that $\rho(a, b)<r$. This implies that $a \in K(b, r)$ and consequently $a \in K(B, r)$. Hence $a \in A \cap K(B, r)$. On the one hand this gives that $A \cap K(B, r) \neq \emptyset$. On the other hand we showed that for any $b \in B$ there is $a \in A \cap K(B, r)$ such that $\rho(a, b)<r$ which means that $b \in K(A \cap K(B, r), r)$ and ends the proof.

In the sequel we show that the order structure of a family $\mathcal{Z}$ generated by the relation of inclusion implies some properties of the function $H_{Z}$.

THEOREM 1. Let $\mathcal{Z}$ be a nonempty subfamily of $\mathcal{M}$ satisfying the condition

$$
X \in \mathcal{Z}, \emptyset \neq Y \subset X \Rightarrow Y \in \mathcal{Z}
$$


Then for any $X \in \mathcal{M}$ the following equality holds

$$
d(X, \mathcal{Z})=D(X, \mathcal{Z}) .
$$

REMARK. Let the family $\mathcal{M}$ be ordered by the inclusion

$$
X \leqslant Y \Leftrightarrow X \subset Y .
$$

Then the condition (1) means that $\mathcal{Z}$ is an initial segment of $\mathcal{M}$ with respect to this order.

Proof: It is obvious that $d(X, \mathcal{Z}) \leqslant D(X, \mathcal{Z})$. In order to show the converse inequality let us take an arbitrary $\varepsilon>0$ and let $r=d(X, \mathcal{Z})$. Then there exists $Z \in \mathcal{Z}$ such that

Hence

$$
d(X, Z)<r+\varepsilon .
$$$$
X \subset K(Z, r+\varepsilon)
$$

which in view of Lemma 1 implies that $Z \cap K(X, r+\varepsilon) \neq \emptyset$ and $X \subset K(Z \cap K(X, r+\varepsilon), r+\varepsilon)$. Consequently

$$
d(X, Z \cap K(X, r+\varepsilon)) \leqslant r+\varepsilon .
$$

On the other hand

$$
Z \cap K(X, r+\varepsilon) \subset K(X, r+\varepsilon)
$$

which allows us to infer that

$$
d(Z \cap K(X, r+\varepsilon), X) \leqslant r+\varepsilon .
$$

Combining (2) and (3) we get

$$
D(X, Z \cap K(X, r+\varepsilon)) \leqslant r+\varepsilon .
$$

But in virtue of the assumption (1) we have that $Z \cap K(X, r+\varepsilon) \in \mathcal{Z}$ so the last inequality implies

$$
D(X, \mathcal{Z}) \leqslant r+\varepsilon .
$$

Taking into account the arbitrariness of $\varepsilon$ we complete the proof.

REMARK. It is easy to show a dual proposition of Theorem 1: if $\mathcal{Z}$ is a nonempty subfamily of $\mathcal{M}$ satisfying the condition

then

$$
X \in \mathcal{Z}, X \subset Y \Rightarrow Y \in \mathcal{Z}
$$

where

$$
\begin{gathered}
D(X, \mathcal{Z})=d(\mathcal{Z}, X) \\
d(\mathcal{Z}, X)=\inf \{d(Z, X): Z \in \mathcal{Z}\} .
\end{gathered}
$$


Corollary 1. Let $\mathcal{Z}$ satisfy the condition (1). If $X \subset Y$ then $H(X) \leqslant H(Y)$.

Proof: By virtue of Theorem 1 it is enough to show that

$$
d(X, \mathcal{Z}) \leqslant d(Y, \mathcal{Z})
$$

Denote $r=d(Y, \mathcal{Z})$. Then for an arbitrary $\varepsilon>0$ there is $Z \in \mathcal{Z}$ such that $d(Y, Z)<$ $r+\varepsilon$ which gives $Y \subset K(Z, r+\varepsilon)$. Consequently $X \subset K(Z, r+\varepsilon)$ which implies $d(X, Z) \leqslant r+\varepsilon$ and ends the proof.

Corollary 2.

(a) $\max \{H(X), H(Y)\} \leqslant H(X \cup Y)$ for all $X, Y \in \mathcal{M}$;

(b) if $X, Y \in \mathcal{M}$ and $X \cap Y \neq \emptyset$ then $H(X \cap Y) \leqslant \min \{H(X), H(Y)\}$.

The proof is a simple consequence of Corollary 1. Moreover, we have

ThEOREM 2. If a family $\mathcal{Z}$ fulfils the condition (1) and the following one also:

$$
A, B \in \mathcal{Z} \Rightarrow A \cup B \in \mathcal{Z}
$$

then

$$
H(X \cup Y)=\max \{H(X), H(Y)\} \text {. }
$$

Indeed, denote $r=\max \{H(X), H(Y)\}$ and take an arbitraiy $\varepsilon>0$. Then there exist $Z_{1}, Z_{2} \in \mathcal{Z}$ such that $X \subset K\left(Z_{1}, r+\varepsilon\right)$ and $Y \subset K\left(Z_{2}, r+\varepsilon\right)$. Hence

$$
X \cup Y \subset K\left(Z_{1}, r+\varepsilon\right) \cup K\left(Z_{2}, r+\varepsilon\right)=K\left(Z_{1} \cup Z_{2}, r+\varepsilon\right)
$$

This implies that $d(X \cup Y, \mathcal{Z}) \leqslant r+\varepsilon$ which in view of Theorem 1 and Corollary 2 completes the proof.

Further, for an arbitrary family $\mathcal{Z} \subset \mathcal{M}$ we will denote

$$
\mathcal{Z}^{0}=\{Z \in \mathcal{Z}: Z \text { is finite }\}
$$

We have

THEOREM 3. If $\mathcal{Z}$ is a family satisfying the condition (1) and $\mathcal{Z} \subset \mathcal{N}$ then

$$
H_{\mathcal{Z}}(X)=H_{Z^{0}}(X)=d\left(X, \mathcal{Z}^{0}\right)
$$

Proof: Keeping in mind Theorem 1 and the inequality $d\left(X, \mathcal{Z}^{0}\right) \leqslant D\left(X, \mathcal{Z}^{0}\right)$ we see that it suffices to prove that $d\left(X, \mathcal{Z}^{0}\right)=d(X, \mathcal{Z})$. Obviously $d(X, \mathcal{Z}) \leqslant d\left(X, \mathcal{Z}^{0}\right)$. Further, let $r=d(X, \mathcal{Z})$. Then, taking an arbitrary $\varepsilon>0$ we can find $Z \in \mathcal{Z}$ such that $d(X, Z)<r+\varepsilon$. Because the set $Z$ is relatively compact, for any $\eta>0$ there 
exists an $\eta$-net $F$ of $Z$. Without loss of generality we may assume that $F \in \mathcal{Z}^{0}$ and even $F \subset Z$. Hence $Z \subset K(F, \eta)$ and consequently

$$
X \subset K(Z, r+\varepsilon) \subset K(K(F, \eta), r+\varepsilon) \subset K(F, r+\varepsilon+\eta)
$$

which gives

$$
d(X, F) \leqslant r+\varepsilon+\eta \text {. }
$$

Taking into account that $\varepsilon$ and $\eta$ were chosen arbitrarily we infer

$$
d\left(X, \mathcal{Z}^{0}\right) \leqslant r,
$$

which completes the proof.

The next property of the function $H_{Z}$ is contained in the following

Theorem 4. If a family $Z$ satisfies the condition (1) then $H(X)=H(X)$ for $X \in \mathcal{M}$.

Proof: According to Theorem 1 we should prove that $d(X, \mathcal{Z})=d(\bar{X}, \mathcal{Z})$. In view of $d(X, Y)=d(X, Y)$, for every $X, Y \in \mathcal{M}$, we obtain $d(X, \mathcal{Z})=d(X, \mathcal{Z})$.

In order to provide further characterisation of the functions $H_{\mathcal{Z}}$ notice that if $\mathcal{Z}$ is a nonempty subfamily of $\mathcal{M}$, for $X, Y \in \mathcal{M}$ and $Z \in \mathcal{Z}$ we have

hence

$$
H_{\mathcal{Z}}(X)=D(X, \mathcal{Z}) \leqslant D(X, Z) \leqslant D(X, Y)+D(Y, Z) ;
$$

and consequently

$$
H_{\mathcal{Z}}(X) \leqslant D(X, Y)+H_{\mathcal{Z}}(Y)
$$

Analogously

$$
\boldsymbol{H}_{\mathcal{Z}}(X)-\boldsymbol{H}_{\mathcal{Z}}(Y) \leqslant D(X, Y) \text {. }
$$

which implies

$$
\begin{aligned}
H_{\mathcal{Z}}(Y)-H_{\mathcal{Z}}(X) & \leqslant D(X, Y) \\
\left|\boldsymbol{H}_{\boldsymbol{Z}}(X)-\boldsymbol{H}_{\boldsymbol{Z}}(Y)\right| & \leqslant D(X, Y) .
\end{aligned}
$$

This means that the function $H_{\mathcal{Z}}$ is continuous with respect to the Hausdorff distance. Thus we obtain

THeOREM 5. $H_{\mathcal{Z}}(X)=0 \Leftrightarrow \bar{X} \in \overline{\mathcal{Z}}$ (the closure of $\mathcal{Z}$ in $\mathcal{M}^{c}$ with respect to the topology generated by $D$ ).

Another property of the function $H$ is given in

TheOREM 6. Assume that $\mathcal{Z}$ satisfies the condition (1) and $\mathcal{Z} \subset \mathcal{N}$. Further, let $X_{n} \in \mathcal{M}^{c}, X_{n} \supset X_{n+1}$ for $n=1,2, \ldots$ and $\lim _{n \rightarrow \infty} H\left(X_{n}\right)=0$. Then the set $X_{\infty}=\bigcap_{n=1}^{\infty} X_{n}$ is nonempty and $X_{\infty} \in \overline{\mathcal{Z}}$.

Proof: Notice that $H\left(X_{n}\right) \geqslant D\left(X_{n}, \mathcal{N}\right)$ which implies that

$$
\lim _{n \rightarrow \infty} D\left(X_{n}, \mathcal{N}\right)=0 .
$$


Thus the thesis is a simple consequence of the properties of Hausdorff measure of noncompactness (see Example 1 below) and Theorem 5.

Finally let us observe that the results contained in Theorems 1-5 show that the function $H_{\mathcal{Z}}$ is a kind of measure of noncompactness defined in an axiomatic way (see $[1,2, \mathbf{3}, \mathbf{6}])$.

We illustrate our investigations by two examples.

EXAMPLE 1: Let $\mathcal{Z}=\mathcal{N}$. Then the function $H$ is called the Hausdorff measure of noncompactness in the space $M$. For its properties we refer to [2].

EXAMPLE 2: Let $\mathcal{Z}$ be the family of all one-point sets in a metric space $M$. Obviously $\mathcal{Z}$ satisfies the condition (1) and $\mathcal{Z} \subset \mathcal{N}$. It is easy to show that in such a case the function $H_{\mathcal{Z}}$ may be expressed in the following way:

where

$$
\begin{gathered}
H_{\mathcal{Z}}(X)=r(X), \\
r(X)=\inf \{\sup \{\rho(x, y): y \in X\}: x \in M\}
\end{gathered}
$$

The number $r(X)$ is called the radius of a set $X$.

\section{REFERENCES}

[1] R.R. Akmerov, M.I. Kamenskii, A.S. Potapov, A.E. Rodkina and B.N. Sadovskii, Measures of noncompactness and condensing operators (Nauka, Novosibinsk, Russian, 1986).

[2] J. Banaś and K. Goebel, Measures of noncompactness in Banach spaces: Lecture Notes in Pure and Applied Mathematics 60 (Marcel Dekker, New York, 1980).

[3] J. Daneš, 'On densifying and related mappings and their applications in nonlinear functional analysis', in Theory of nonlinear operators, pp. 15-56 (Akademic-Verlag, Berlin, 1974).

[4] D.M. Duc, 'Measure of noncompactness and spectral theory', Math. Nachr. 115 (1984), 305-317.

[5] K. Kuratowski, Topology (Academic Press, 1966).

[6] A. Martinon, 'A system of axioms for measures of noncompactness', Extracta Math. 4 (1989), 44-46.

[7] L.W. Weis, 'On the computation of some quantities related to semi-Fredholm operators', in Proc. 12th Winter School on Abstract Analysis (Srni, Bohemian Forest, 1984).

Department of Mathematics Technical University of Rzeszow 35-959 Rzeszow, W. Pola 2

Poland
Department of Mathematical Analysis

University of La Laguna

38271 La Laguna (Tenerife)

Spain 